\title{
Preface: Immunological Tango in Barcelona 2011
}

\author{
Sudhir Gupta • Hans Peter Schwarz
}

Received: 16 July 2012 / Accepted: 24 July 2012 /Published online: 10 August 2012

(C) Springer Science+Business Media, LLC 2012

In recent years there have been major developments in the understanding of the mechanisms of action of immunoglobulins and natural antibodies, and in the pathogenesis of a number of neurological diseases. Furthermore, there has been a renewed interest in one of the oldest cytokines, the migration inhibition factor (MIF), for its role in inflammation and cancer. These were among the topics discussed at the second International Forum on Immunoglobulin Research (IFIR) held in Barcelona, Spain, on September 1417, 2011. Both IFIR and this supplement were sponsored by Baxter Healthcare Corporation.

Immunoglobulins have both anti-inflammatory and proinflammatory effector properties that have been shown for both $\operatorname{IgA}$ and $\operatorname{IgG}$ isotypes. These contrasting properties make them attractive tools for the development of therapeutic approaches. Falk Nimmerjahn (Erlangen, Germany) discussed the use of animal model systems in understanding the mechanisms of $\operatorname{IgG}$ activities, and highlighted the differences in $\operatorname{IgG}$ subclasses and FcR between humans and even closely related monkeys. Because these differences limit extrapolation of data from monkeys to humans, he discussed various alternative animal model systems to study the mechanisms and limitations of therapeutic antibodies. These models include transgenic mice expressing human proteins regulated by their endogenous promoters. Immunodeficient mice like Rag2, SCID, or NOD/SCID are injected with human

\footnotetext{
S. Gupta $(\bowtie)$

Division of Basic and Clinical Immunology,

University of California,

Irvine, CA, USA

e-mail: sgupta@uci.edu

H. P. Schwarz

Wagramer Strasse 17-19,

1221 Vienna, Austria

e-mail: hans_peter_schwarz@baxter.com
}

peripheral blood mononuclear cells, followed by injection of therapeutic antibodies. Another model, which appears to be the most desirable, transplants immunodeficient mice with human hematopoietic stem cells.

Francis William Rogers Brambell, an Irish zoology professor, observed that passive transfer of immunity via gamma globulin occurred from mother to offspring, and subsequently hypothesized the existence of a receptor that can mediate the transfer of maternal gamma globulin to infant as well as the protection of gamma globulin from catabolism. Neil Simister and Keith Mostov purified and sequenced an Fc receptor from the intestine of an 11-day-old rat. The receptor, hence named neonatal FcR, was associated with $\beta 2$-microglobulin, which is structurally similar to the MHC I molecule. In 1994, Pam Bjorkman and colleagues reported on the crystal structure of FcRn. FcRn is evolutionarily distinct from other FcRs and is expressed on a variety of tissues and cells. Richard Blumberg (Boston, MA, USA) discussed expression and functions of FcRn in adults at the level of the vascular endothelium, where it protects IgG from catabolism. Also, antigen-presenting cells use FcRn to recycle internalized IgG rather than destroy it. At the cell surface, Fc $\gamma$ IIR would bind immune complexes; once internalized, immune complexes could be transferred to FcRn in the endosome and then returned to the cell surface, where they would be released at physiological $\mathrm{pH}$ for cross-presentation. Dr Blumberg also discussed engineering FcRn to increase binding of FcRn to IgG, which may be exploited for therapeutic purposes.

Naïve $\mathrm{CD}^{+} \mathrm{T}$ cells differentiate into IL-17-producing Th17 cells, in which ROR $\gamma \mathrm{t}$ (RORC) transcription factor plays a critical role. IL-17 plays an important role in protecting against extracellular microbes, especially fungi. However, increased IL-17 is associated with inflammation and autoimmunity. Jagadeesh Bayry (Paris, France) discussed the effect of intravenous immunoglobulin (IVIG), which could be more appropriately termed immunoglobulin $G$ 
( $\mathrm{IgG})$, especially for its in vitro effects; IgG is administered by both intravenous and subcutaneous routes, on Th17 cells. He demonstrated that IgG suppresses differentiation, amplification, and functions of human Th17 cells, and these suppressive effects were mediated by inhibiting phosphorylation of transcription factor RORC and STAT-3 signaling, and reciprocal regulation of Treg. Furthermore, these effects were not due to anti-IL-17 antibodies in immunoglobulin preparations used.

In recent years, properties of anti-Fas-specific, sialylated, and recombinant sialylated $\mathrm{IgG}$ preparations have been reported. Yehuda Shoenfeld (Tel Aviv, Israel) discussed molecular targeting of autoimmune processes using specific IVIG preparations. Using a peptide phage display library technology, he and his associates identified a panel of lupusrelated synthetic idiotypes. A column composed of these synthetic lupus-related idiotypes was used to prepare a large amount of lupus-specific IVIG preparations. These preparations were 200 times more beneficial in autoimmune NZB/ W F1 mice models than regular IVIG. Dr Shoenfeld also presented data on the beneficial effect of anti-beta-2-glycoprotein-I ( $\beta 2 \mathrm{GPI})$-specific IVIG in patients with antiphospholipid syndrome (APS). This APS-specific IVIG reduced the fetal loss induced by anti- $\beta 2$ GPI antibodies by improving the implantation process in a mouse model. IVIG specific for anti-desmoglein $1+3$ antibodies prevented the development of experimental pemphigus vulgaris, and IVIG specific for acetylcholine-receptor antibodies ameliorated the development of experimental myasthenia gravis.

Anne De Groot (Providence, RI, USA) discussed Tregitopes as a novel tool for tolerance induction and treatment of autoimmunity. Tregitopes are highly conserved T cell epitopes in Fc and are immediately adjacent to the hypervariable domains of IgG (Fab) that induce T cells with immunoregulatory properties (Treg). Natural regulatory T cell (nTreg) epitopes are present in immunoglobulins. She reported an antigen-specific immunosuppressive effect of Tregitopes and also described 5 well-characterized Tregitopes, which bind to both mouse and human MHC. The beneficial results of Tregitopes in various animal models of allergic and autoimmune disease also were discussed.

Ricardo Sorensen (New Orleans, LA, USA) discussed specific antibody deficiency syndrome (SAD), which is classically defined as a syndrome characterized by failure to mount a specific antibody response to Pneumovax 23 in the presence of normal IgG, IgG subclasses, IgM, and IgA. However, SAD may be present in a variety of primary immunodeficiency diseases. Dr Sorensen discussed SAD in response to Pneumovax 23 as well as to conjugate pneumococcal polysaccharide vaccine (PCV-SAD) in relation to infections and B cell subsets. Switched memory B cells were significantly decreased in SAD compared with controls and PCV-SAD. However, a more detailed study is needed before switched memory B cells could be used as one of the immunological markers for SAD, especially when such alterations in these B cells are observed in other primary immunodeficiency diseases.

Natural antibodies/autoantibodies are predominantly of the $\operatorname{IgM}$ isotype that are polyspecific and bind to polysaccharide antigens with low affinity. They play an antimicrobial, housekeeping, and homeostatic role. Natural IgMs show potential for the treatment of neurologic diseases. Moses Rodriguez (Rochester, MN, USA) discussed recent data supporting a role for natural autoantibodies in remyelination and axon protection. His group identified a natural human monoclonal IgM that binds to the surface of neurons in vitro and promotes neurite outgrowth. They generated a fully human recombinant form (rHIgM12) with properties identical to serum-derived IgM. Dr Rodriguez discussed their data on the mechanism of rHigM12-mediated process outgrowth in primary hippocampal neurons and whether the IgM had a therapeutic effect in Theiler's virus-infected SJL mice, a model of MS. They observed that rHIgM12 recruits membrane molecules such as cholesterol and GM1 to IgMneuronal membrane contact sites affecting microtubule dynamics to drive axon extension. This recruitment may activate downstream signals for axonal specification. They proposed that pentameric IgM structure is critical to cluster membrane lipids and proteins, which results in signal cascades. Peripheral treatment with the same antibody protects spinal cord axons in vivo and also influences functional motor improvement.

Neuromyelitis optica (NMO) is an idiopathic central nervous system inflammatory demyelinating disease that causes optic neuritis and transverse myelitis. It is associated with autoantibodies that target the astrocyte water channel aquaporin-4. Evidence exists to support autoimmune pathogenesis of NMO. Dean Wingerchuk (Scottsdale, AZ, USA) reviewed the nosology, pathobiology, and clinical course of NMO, and discussed the rationale and potential role for IVIG in 2 current therapeutic strategies for NMO: relapse treatment and relapse prevention. IVIG may be considered in patients with NMO where current therapies are contraindicated or relapses of NMO are resistant to therapy. However, randomized controlled trials are needed.

Multifocal motor neuropathy (MMN), a rare inflammatory neuropathy, is characterized by slowly progressive asymmetric distal limb weakness without sensory loss. Leonard van den Berg (Utrecht, the Netherlands) reported on a cross-sectional study of the clinical characteristics, outcome, and pathophysiology of all patients with MMN in the Netherlands. Initial diagnosis was motor neuron disease in onethird of patients, and typical age at onset in men was younger than in women ( 35 vs. 45 ). Conduction blocks were most frequently detected in the ulnar $(80 \%)$ and median (77\%) nerves. Anti-GM1 antibodies were found in $43 \%$. 
Higher antibody titers correlated with more severe weakness. Ninety-four percent of the patients responded to IVIG. Determinants of more severe weakness and disability were the number of years without IVIG treatment and the amount of axon loss. Results of this study underscore the importance of early IVIG treatment to prevent axonal degeneration.

High-dose IVIG is used for the treatment of several neurological autoimmune diseases. Although efficacy has been shown in randomized clinical trials in neurological autoimmune diseases (AID), Guillain-Barré syndrome (GBS), chronic inflammatory demyelinating polyneuropathy (CIDP), and MMN, long-term outcome data on drug utilization, effectiveness, tolerability, health-related quality of life (HRQoL), and economic variables are lacking. Martin Stangel (Hannover, Germany) discussed interim analysis from a prospective, observational, and Internet-based registry. Quality of life in AID patients is substantially compromised. However, larger patient numbers and extended follow-up periods will be required.

Schwann cells, the myelinating glial cells of the peripheral nervous system (PNS), establish lipid-rich myelin sheaths around axons and enable saltatory nerve conduction via axonal voltage-gated sodium channels concentrated at narrow gaps (nodes of Ranvier). Schwann cells also contribute to peripheral nerve regeneration when axons are damaged. Fully differentiated Schwann cells can dedifferentiate, then re-enter the cell cycle and redifferentiate to produce new myelin sheaths around regenerating axons. Developmental differentiation as well as regenerative de- and redifferentiation is tightly regulated by a balance between positive and negative regulators of Schwann cell maturation. Patrick Küry (Düsseldorf, Germany), reviewed data of complex interactions between extrinsic and intrinsic signals that inhibit Schwann cell differentiation. In particular, he discussed a role of p57kip2, an intrinsic inhibitor of myelinating glial cell differentiation.

An entire session was devoted to the biology of MIF, one of the first cytokine activities described by John David in 1966. However, MIF was not cloned and characterized until the 1990s. MIF appears to maintain cellular activation by counter-regulating the immunosuppressive action of glucocorticoids and by inhibiting stimulus-induced apoptosis. MIF binds to an extracellular domain of CD74 and mediates signals via CD74 association with CD44. The role of MIF was discussed in relation to inflammation, cancer, and autoimmunity.

Thorsten Hagemann (London, UK) reviewed the role of inflammatory cytokines in cancer, and discussed in detail the role of MIF in cancer. MIF has been implicated in the angiogenesis switch of early cancer. More recently MIF has been shown to be a potential ligand for CXCR2 and CXCR4, both of which appear to play an important role in ovarian cancer. Though inflammatory cells may produce growth factors for tumor cells, identifying the mechanisms by which inflammation is dysregulated, as well as how inflammation provides tumor-promoting signals, may offer a novel therapeutic approach for cancer.

The discovery of functional alleles for MIF and their association with various rheumatological disorders has prompted renewed interest in the role of MIF in immunopathogenesis, and in pharmacogenomic approaches to regulate MIFdependent immune pathways. Richard Bucala (New Haven, CT, USA) reviewed MIF genetics, the role of MIF in autoimmunity, and MIF alleles in systemic lupus erythematosus (SLE). He discussed results from a study analyzing MIF polymorphisms in a cohort of SLE patients, and related mouse modeling studies suggesting that MIF has a dual role in the immunopathogenesis of human SLE. Unexpectedly, reduced susceptibility to SLE was associated with high expression $M I F$ alleles, perhaps through a mechanism involving enhanced clearance of pathogens. Among SLE patients with nephritis, serositis, and CNS involvement, the study found reduced frequencies of low-expression MIF genotypes compared with patients without these sequelae. This suggests that low-expression MIF alleles may protect against inflammatory end-organ damage. Humanized anti-MIF receptors and antiMIF antibodies currently are in clinical development, and may offer optimism for the treatment of SLE and other autoimmune diseases.

In the next few years, it is likely that the role of antibodies and soluble mediators will be better defined. These include chemokines and cytokines in the pathogenesis of a variety of neurological disorders, many of which were discussed at IFIR 2011. Furthermore, antibodies and soluble mediators might be used as therapeutic targets for various autoimmune and inflammatory disorders. 